Regular Paper

\title{
Proposal of Load and Amplitude Estimation Method for Linear Resonant Actuator Using Two Back-EMF Signals
}

\author{
Masayuki KATO*1 $^{* 1}$ Stu. Mem.), Katsuhiro HIRATA*1 (Mem.), Yasuyoshi ASAI ${ }^{* 1}$ and Takamichi YOSHIMOTO*1
}

\begin{abstract}
Linear resonant actuators (LRAs) have been used and studied because of high efficiency. However, the amplitude of LRAs severely decreases when external loads are applied. As a solution to this problem, we have proposed an external load estimation method using two signals of the back-EMF. However, the proposed method was effective only under unipolar drive. This paper proposes load and amplitude estimation method for LRAs using two back-EMF signals under bipolar PWM feedback control. The effectiveness of this method was verified through 2-D FEM analysis of our code.
\end{abstract}

Keywords: linear resonant actuator, load estimation, amplitude estimation, finite element method. (Received: 14 December 2014, Revised: 23 February 2015)

\section{Introduction}

Linear oscillatory actuators (LOAs) have been used in a wide range of applications [1-5] because they can reciprocate in a comparatively short stroke in spite of their compact size and light weight. Especially, linear resonant actuators (LRAs) have been used and studied $[6,7]$ because the resonance causes high efficiency. However, the amplitude of LRAs severely decreases when external loads are applied. With applications such as electric shaver or air compressors, the amplitude of the mover needs to be able to be arbitrarily controlled according to the external load.

We applied PID control to an LRA and the mover amplitude remained constant against an external load [8, 9]. Furthermore, we proposed an external load estimation method using two signals of the back-EMF [10]. As a result, it became possible to estimate the external load, and it was possible to obtain an arbitrary amplitude by changing the target voltage of PID control according to the estimated load [11]. However, the proposed method is effective only under unipolar drive.

In this paper, we propose load and amplitude estimation methods for an LRA using two back-EMF signals under bipolar drive. The effectiveness of these methods is verified through 2-D finite element method (FEM) analysis [12-14] in which the magnetic field equation was coupled with the electric circuit equation, control method, and motion equation. Also, measurements were performed to verify the effectiveness of the proposed method.

\section{Basic Structure and Operating Principle}

\footnotetext{
Correspondence: Masayuki KATO, Department of Adaptive Machine Systems, Graduate School of Engineering, Osaka University, 2-1, Yamadaoka, Suita, Osaka 565-0871, Japan email: masayuki.kato@ams.eng.osaka-u.ac.jp

${ }^{*}$ Osaka University
}

The basic structure of the LRA in this study is shown in Fig.1. This actuator mainly consists of two movers, a common stator and resonant springs that support the mover to maintain the air-gap length $(0.36 \mathrm{~mm})$. Two parallelly arranged movers are composed of two opposite pole magnets fixed on a back yoke. The common stator is composed of an E-shaped laminated yoke with an excitation coil of 68 turns at its middle pole.

Fig. 2 shows the operating principle. The magnetic poles of the stator are magnetized when the coil is excited, and thrust is generated. Two movers move in the opposite direction of each other by exciting the coil. The operation frequency of the LRA is substantially determined by the spring constant and the mass of the mover. This actuator is operated under PWM feedback control, and excitation time (duty) is decided by detecting the back-EMF while the coil is not excited.

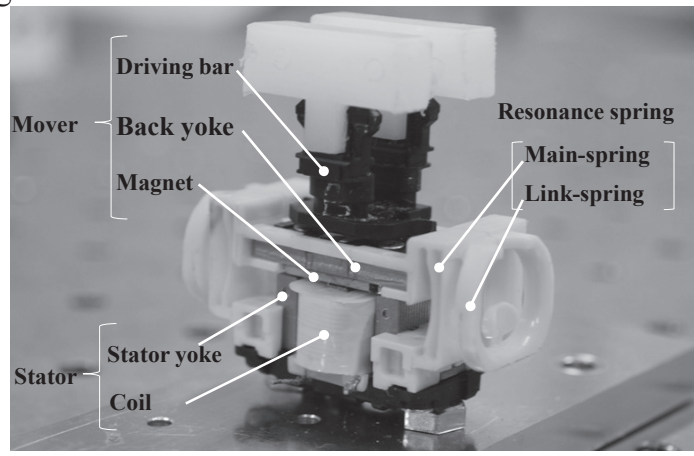

Fig. 1. Basic structure of the LRA.
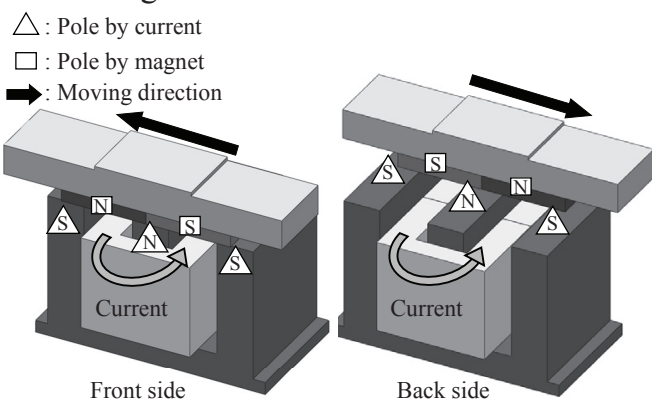

Fig. 2. Operating Principle. 


\section{Control Method and Load Estimation Method}

\subsection{PWM feedback control}

Fig. 3 shows the bipolar PWM feedback control of this actuator. The circuit diagram of this operating mode is shown in Fig. 4. First, in interval a, the coil detects the back-EMF $V_{1}$ after a positive-going zero crossing. In interval $b$, the coil is excited with a delayed time $(25 \mu \mathrm{s})$. In interval $\mathrm{c}$, the coil is excited under PWM control according to the duty determined by PID control using the back-EMF $V_{1}$. The circuit diagram of this operating mode is shown in Fig. 4(a). In interval d, current circulates through a diode as shown in Fig. 4(b). Finally, in interval e, the circuit is opened as shown in Fig. 4(c). It is highly effective to drive the actuator under advanced PWM control, in which a multiple square wave voltage is applied, and the actuator can be operated under low electric power because the current does not decrease immediately even if the input voltage is turned off.

In interval $\mathrm{c}$, the coil is excited according to the duty determined by PID control using the back-EMF $V_{1}$. The duty is defined by equations (1) and (2):

$$
\begin{gathered}
\operatorname{Duty}(\%)=K_{p} e(t)+K_{I} \int e(t) d t+K_{D} \frac{d e(t)}{d t} \\
e(t)=V_{S}-V_{1}
\end{gathered}
$$

Where $K_{\mathrm{P}}$ is the proportional gain, $K_{\mathrm{I}}$ is the integral gain, $K_{\mathrm{D}}$ is the differential gain, $e(\mathrm{t})$ is the deviation, and $V_{\mathrm{S}}$ is the target voltage.

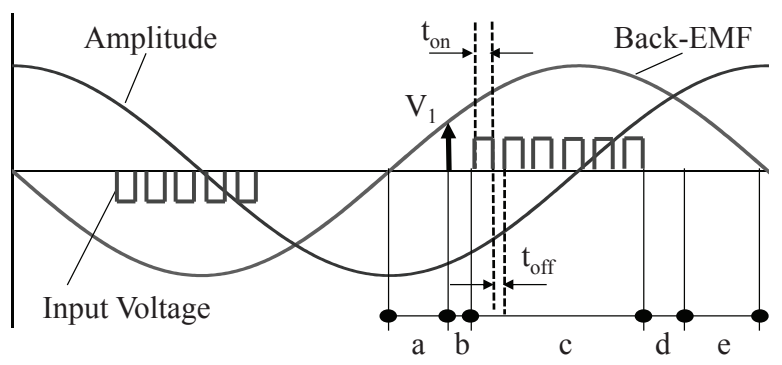

Fig. 3. PWM feedback control.

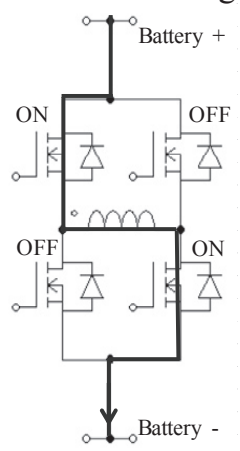

(a) Excitation mode

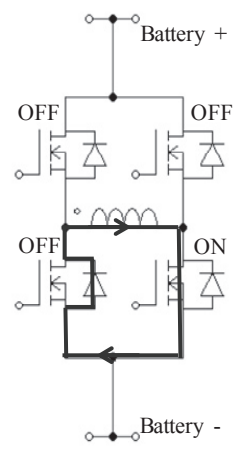

(b) Circulation mode

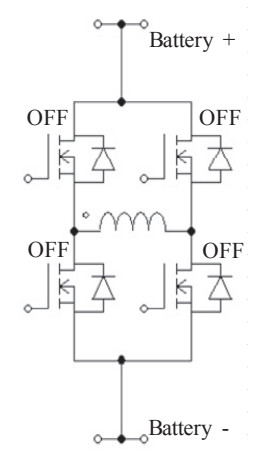

(c) Open mode
Fig. 4. Control circuit.

\subsection{New load estimation method for bipolar drive}

Previously, we have established an external load estimation method and found it to be effective. However, the proposed method was effective only under unipolar PWM feedback control. In addition, bipolar drive is superior to unipolar drive because of its higher efficiency and larger amplitude. Therefore, an external load estimation method under bipolar PWM feedback control is also needed.

Fig. 5 shows a schematic diagram of the load estimation method. The mover is operated at the frequency of about $220 \mathrm{~Hz}$. We propose a new load estimation method under bipolar PWM feedback control using two backEMF signals: $V_{\mathrm{A}}$ and $V_{\mathrm{B}} . V_{\mathrm{A}}$ is the value when a constant time $(1600 \mu \mathrm{s})$ has passed after a negative-going zero crossing of the back-EMF. $V_{\mathrm{B}}$ is the value when a constant time $(250 \mu \mathrm{s})$ has passed after a positive-going zero crossing. $V_{\mathrm{B}}$ is the same value as $V_{1}$ shown in Fig. 3. As shown in Fig. 5, the mover is in the free damped oscillation when the PWM voltage is not applied. Then, the load estimation function is formulated by solving motion equations under the damped oscillation. First, when the mover velocity is negative, the motion equation is expressed as equation (3):

$$
M \frac{d^{2} x}{d t^{2}}+K x=+F
$$

Where $M$ is the mass of mover, $x$ is the displacement of the mover, $K$ is the spring constant, $F$ is the external load. Equation (4) is obtained by solving equation (3) and substituting the initial value. Equation (5) is obtained by differentiating equation (4):

$$
\begin{gathered}
x=\left(x_{0}-\alpha\right) \cos \omega_{n} t+\alpha \\
v=-\omega_{n}\left(x_{0}-\alpha\right) \sin \omega_{n} t \\
\omega_{n}=\sqrt{\frac{K}{M}} \\
\alpha=\frac{F}{K}
\end{gathered}
$$

Where $x_{0}$ is the initial position shown in Fig. 5 and $\omega_{\mathrm{n}}$ is the angular resonant frequency. Next, when the mover velocity is positive, the motion equation is expressed as equation (8):

$$
M \frac{d^{2} x}{d t^{2}}+K x=-F
$$

Equation (9) is obtained by solving equation (8) and considering the continuity of its motion. Equation (10) is obtained by differentiating equation (9):

$$
\begin{aligned}
& x=\left(x_{0}-3 \alpha\right) \cos \omega_{n} t-\alpha \\
& v=-\omega_{n}\left(x_{0}-3 \alpha\right) \sin \omega_{n} t
\end{aligned}
$$



(10):

Equation (11) is obtained from equations (5) and

$$
\alpha=\frac{F}{K}=\frac{1}{2}\left(\frac{v_{B}}{\omega_{n} \sin \omega_{n} t_{B}}-\frac{v_{A}}{\omega_{n} \sin \omega_{n} t_{A}}\right)
$$

Therefore, the load estimation function can be obtained as shown in (12):

$$
F^{\prime}=\frac{K}{2}\left(\frac{K_{E} V_{B}}{\omega_{n} \sin \omega_{n} t_{B}}-\frac{K_{E} V_{A}}{\omega_{n} \sin \omega_{n} t_{A}}\right)
$$

Where $F^{\prime}$ is the estimated load, $K_{E}$ is the back-EMF constant. In equation (12), $K_{E}$ is regarded as constant and detent force is regarded as a linear spring. However, $K$ and $K_{E}$ are dependent on the mover position as shown in Fig. 6. Therefore, these values need to be amended according to the mover position. Then, from this, the estimated load can be corrected by using the amplitude estimation function shown in (13):

$$
x_{1}^{\prime}=\frac{1}{2}\left(\frac{K_{E} V_{B}}{\omega_{n} \sin \omega_{n} t_{B}}+\frac{K_{E} V_{A}}{\omega_{n} \sin \omega_{n} t_{A}}\right)
$$

Where $x_{1}$ ' is the estimated amplitude minimum value of the mover position. Fig. 7 shows the flowchart for the load correction method. Functions $g$ and $h$ were calculated from the static analysis result.

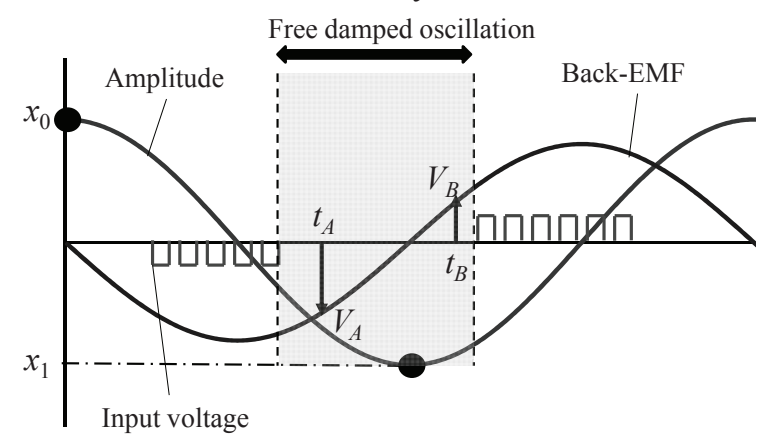

Fig. 5. Load estimation method.

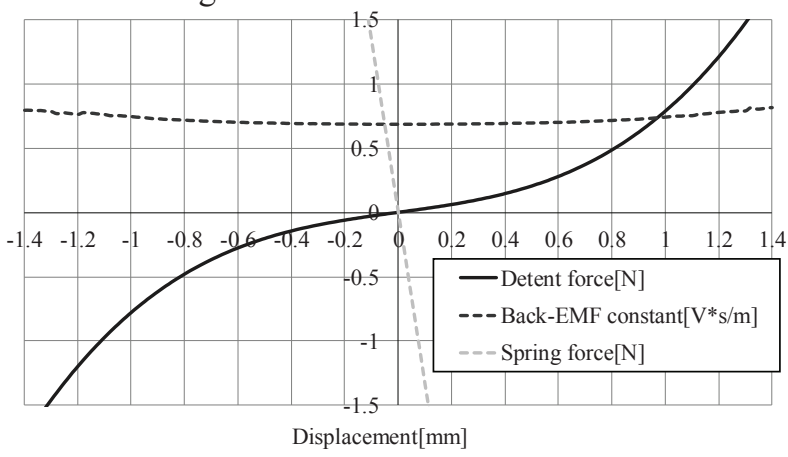

Fig. 6. Nonlinear detent force and back-EMF constant.

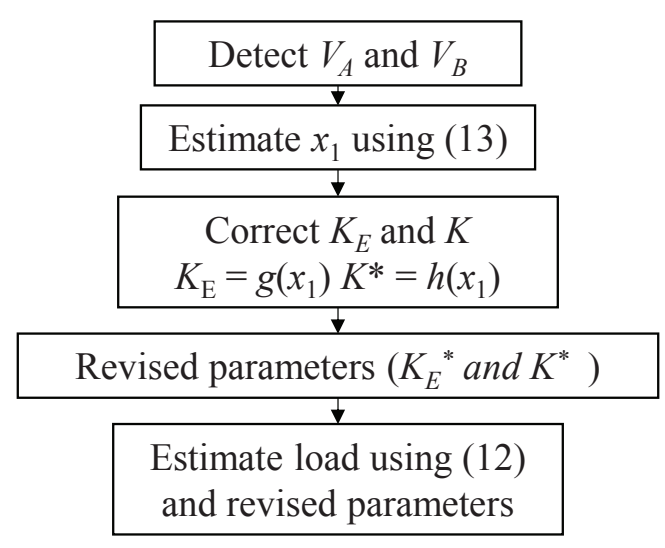

Fig. 7. Flowchart for load correction method.

\section{Analysis Method}

\subsection{Coupling Analysis with Motion Equation}

The motion equation is given as follows:

$$
M \frac{d^{2} x}{d t^{2}}+C \frac{d x}{d t}+K x \pm F=F_{x}
$$

Where $C$ is the viscous damping coefficient and $F_{\mathrm{x}}$ is the thrust of the mover. The thrust of the mover is calculated using the Maxwell stress tensor method, and is substituted into the equation (14). The position of the mover is calculated at each time step. Fig. 8 shows the flowchart for this coupled analysis.

\subsection{Analysis Model and Condition}

Fig. 9 shows the 2-D FEM model. In this paper, 2-D FEM analysis is performed because the mover shape is uniformed to depth direction and the mover has two dimensional distribution of magnetic flux. The number of elements is about 19,000 , and the unknown variables are about 9,500. Table 1 shows the analysis conditions. The resistance of the circuit for when it is in excitation mode and circulation mode are $350 \mathrm{~m} \Omega$ and $290 \mathrm{~m} \Omega$ respectively. The number of steps is 15,000 , time division is $10 \mathrm{~ms}$, and total computation time is about 3 hours.

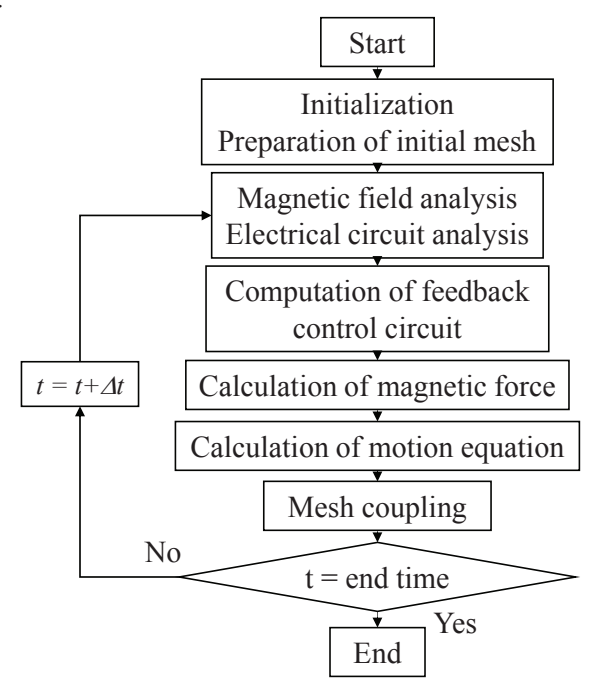

Fig. 8. Flowchart for analysis. 


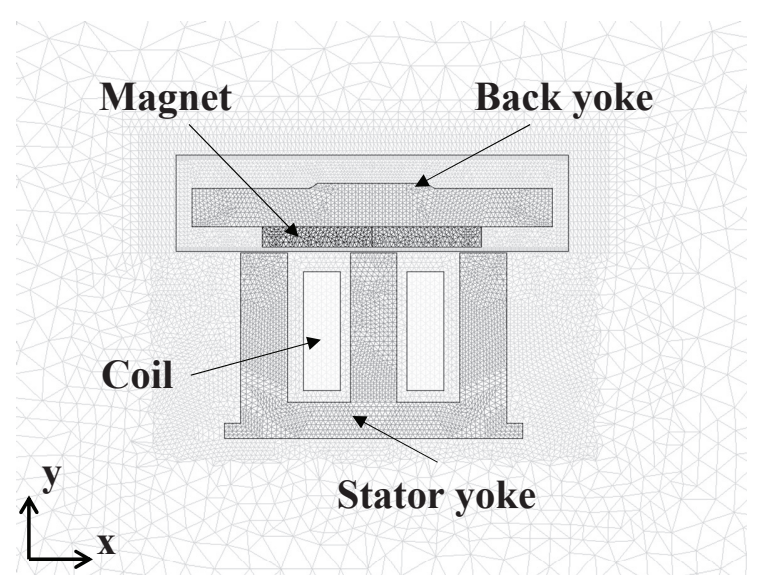

Fig. 9. 2-D FEM model.

Table 1. Analysis condition.

\begin{tabular}{c|c|c}
\hline \multirow{4}{*}{ Coil } & Input voltage $(\mathrm{V})$ & 3.6 \\
\cline { 2 - 3 } & Resistance $($ on) $(\mathrm{m} \Omega)$ & 350 \\
\cline { 2 - 3 } & Resistance $(\mathrm{off})(\mathrm{m} \Omega)$ & 290 \\
\cline { 2 - 3 } & Number of turns $(\mathrm{turn})$ & 68 \\
\hline Magnetization of magnets $(\mathrm{T})$ & 1.42 \\
\hline Mass of mover $(\mathrm{g})$ & 6.95 \\
\hline \multicolumn{2}{c}{ Viscous damping coefficient $(\mathrm{N} \cdot \mathrm{s} / \mathrm{m})$} & 0.14 \\
\hline Spring constant $\mathrm{N} / \mathrm{mm})$ & 0.5 \\
\hline
\end{tabular}

\section{Dynamic Analysis under Proposed Method}

Fig. 10 shows the analyzed result of the estimated steady-state amplitude when an external load was applied to the LRA. In this analysis, the target voltage $V_{\mathrm{S}}$ was adjusted according to the different loads. From Fig. 10, the estimated values agree with the analysis value. Fig. 11 compares the steady-state estimated results before and after applying the correction method. From Fig. 11, it is observed that the load correction method is effective and the external load under bipolar PWM feedback control can be estimated at various values of the amplitude although the estimation error increases under higher amplitude.

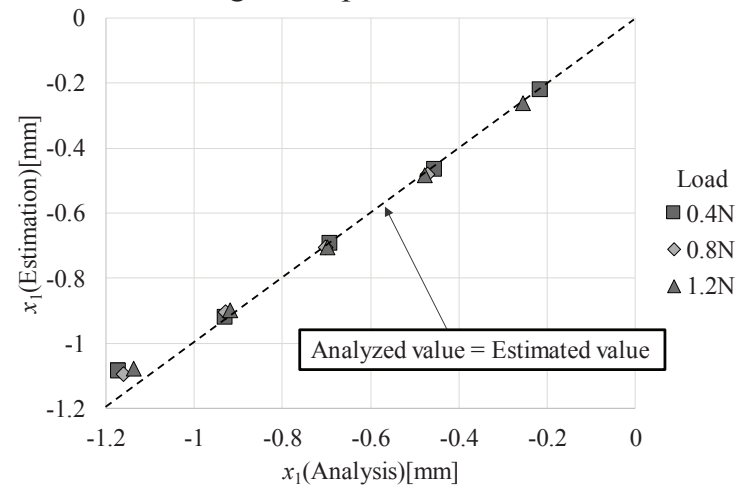

Fig. 10. Analyzed results of estimated amplitude.

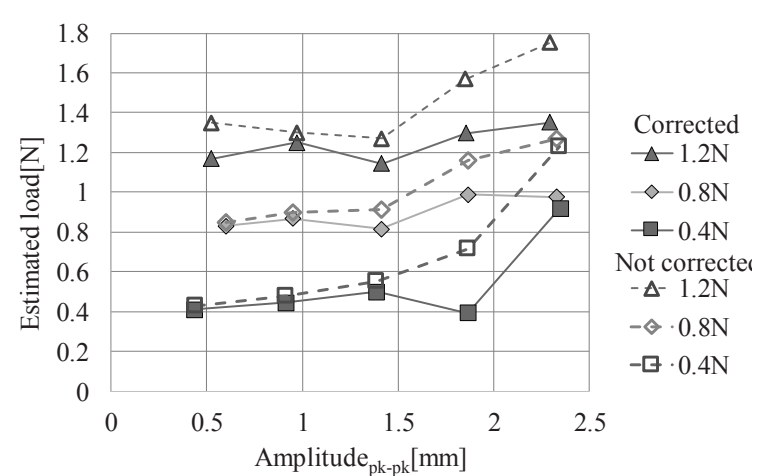

Fig. 11. Comparison between two results.

\section{Experiment under Proposed Method}

\subsection{Measuring System}

Measurements were performed to verify the effectiveness of this new control method. Experimental setup of dynamic characteristics measurement is shown in Fig. 12. A direct voltage of $3.6 \mathrm{~V}$ supplied from a stabilized power supply was converted into the PWM voltage with the control circuit controlled by a microcomputer, and was applied to the coil.

Fig. 13 shows the experimental setup. Since applying a horizontal load is difficult, we instead applied a vertical load and later calculated it to its horizontal load equivalent. The amplitude of the mover and the backEMF $V_{\mathrm{A}}$ and $V_{\mathrm{B}}$ were measured when the vertical load (weight) was varied from $750 \mathrm{~g}$ to $2000 \mathrm{~g}$. The coefficient of friction was measured to be 0.06 .

\subsection{Experimental Results}

Fig. 14 shows the measured result of the estimated steady-state amplitude when an external load was applied to the LRA. In this experiment, the target voltage $V_{\mathrm{S}}$ was adjusted according to the different loads. From Fig. 14, it was verified that the amplitude estimation method was effective since the measured value and the estimated values agreed with each other.

Fig. 15 shows the measured result of the estimated steady-state load. We can see that the estimated values are severely different from the actual values. These errors are caused by mainly three reasons. First, This LRA has two movers. Therefore, the stable and equal load is not applied to the LRA. Second, as shown in Fig. 16 , the back-EMF is noisy. Third, in equations (3) and (8), viscous force is not considered because its value is thought to be smaller than that of the external load. However, in current measurement, the viscous force is thought to be too big to approximate.

In the near future, we will improve the load device so that external load can be equal and constant and install a digital filter to remove the noise. 


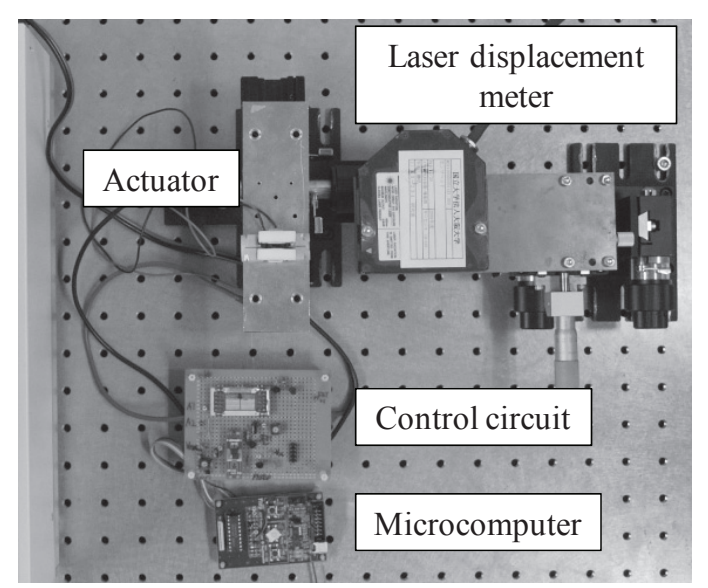

Fig. 12. Experimental setup of dynamic characteristic measurement.

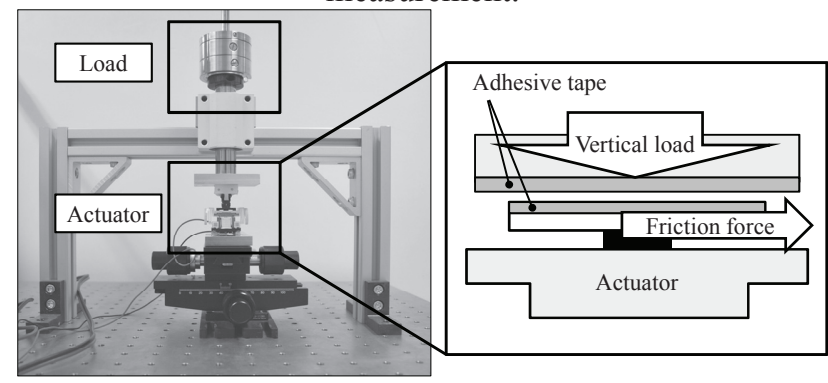

Fig. 13. Load device.

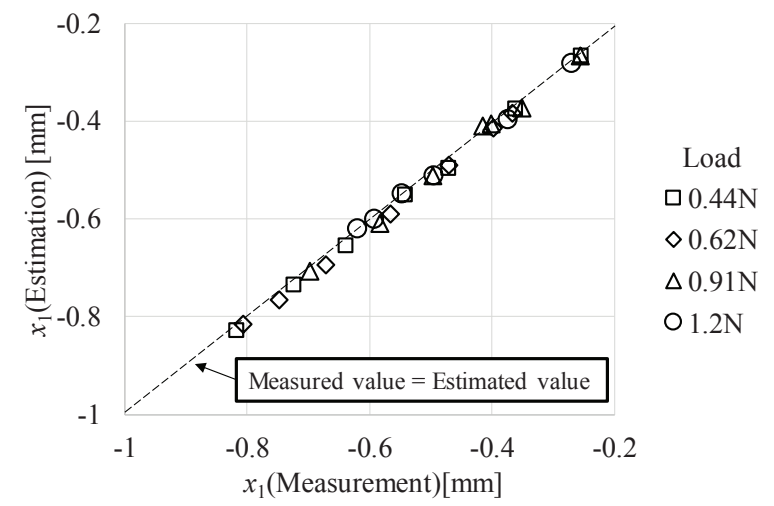

Fig. 14. Measured results of estimated amplitude.

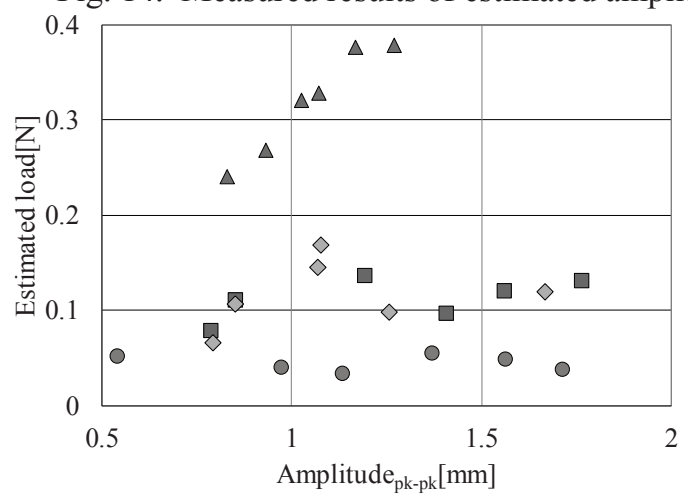

Load

$00.44 \mathrm{~N}$

$\square 0.62 \mathrm{~N}$ $\diamond 0.91 \mathrm{~N}$ $\Delta 1.2 \mathrm{~N}$

Fig. 15. Measured results of estimated load.

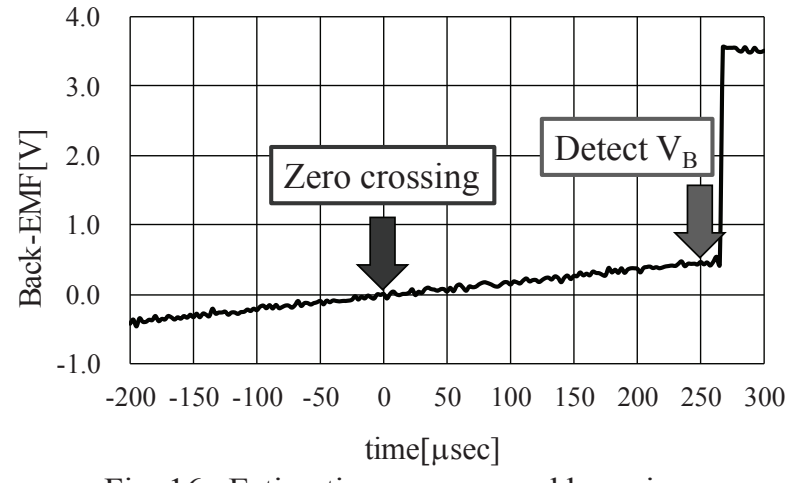

Fig. 16. Estimation error caused by noise.

\section{Conclusion}

This paper presented an external load estimation method for our LRA using two signals of the back-EMF under bipolar PWM feedback control. The effectiveness of this method was determined through 2-D FEM analysis, in which the magnetic field equation was coupled with the electric circuit equation, control method, and motion equation. It was found that it was possible to estimate the load under bipolar PWM feedback control.

Measurements were performed to verify the effectiveness of this proposed method. It was confirmed in the measurements that the amplitude estimation method was effective. However, the estimated load did not agree with the actual values because of the unstable load device, noise of back-EMF, and too big viscous force in current experiment system.

In the near future, we will improve the load device so that external load can be equal and constant and install a digital filter to remove the noise.

In addition, we will establish an amplitude control method under bipolar PWM feedback control.

\section{References}

[1] K. Hirata, T. Yamamoto, T. Yamaguchi, Y. Kawase and Y. Hasegawa, "Dynamic Analysis Method of TwoDimensional Linear Oscillatory Actuator Employing Finite Element Method", IEEE Trans. Magn., vol.43, No.4, pp.1441-1444, 2007.

[2] J. Wang, Z. Lin, D. Howe, "Characteristics of Linear Compressors under Current Source Excitation”, Proceedings of the Institution of Mechanical Engineers, Part A: Journal of Power and Energy November 1, 2007 vol. 221 no. 7 1057-1065

[3] M. Watada, K. Yanashima, Y. Oishi, D. Ebihara and H. Dohmeki, "Improvement of characteristics of linear oscillatory actuator for artificial hearts", IEEE Trans. Magn., vol.29, No.6, pp.3361-3363, 1993.

[4] Tae Heoung Kim, Hyung-Woo Lee, Youn-Hyun Kim, Ju Lee, "Development of a Flux Concentration-Type Linear Oscillatory Actuator", IEEE Trans. Magn., vol.40, No.4, pp.2092-2094, 2004.

[5] D. Howe and J. Wang., "A Novel Linear Drive for Nextgeneration Reciprocating Vapor Compressors", IEEJ Trans. Elec Electron Eng., vol.3, No.5, pp.455-43, 2008. 
[6] Y. Asai, K. Hirata, and T. Ota, "Dynamic Analysis Method of Linear Resonant Actuator with Multi-Movers Employing 3-D Finite Element Method", IEEE Trans. Magn., vol.46, no.8, pp.2971-2974, Aug. 2010.

[7] T. Yamaguchi, Y. Kawase, S. Suzuki, K. Hirata, T. Ota and Y. Hasegawa "Dynamic Analysis of Linear Resonant Actuator Driven by DC Motor Taking into Account Contact Resistance between Brush and Commutator", IEEE Trans. Magn., vol.44, No.6, pp.1510-1513, Jun. 2008.

[8] K. Matsui, K.Hirata, and T.Ota, "Dynamic Analysis of Linear Resonant Actuator under PWM Control Employing the 3-D Finite Element Method", Proceedings of the 13th Biennial IEEE CEFC (Conference on Electromagnetic Field Computation), OC1-1, p.192, 2008.

[9] Y. Asai, K. Hirata, and T. Ota, "3-D Finite Element Analysis of Linear Resonance Actuator under PID Control", Proceedings of the 14th Biennial IEEE CEFC (Conference on Electromagnetic Field Computation), 32P8, 2010.

[10] Y. Asai, K. Hirata and T. Ota, "PID Feedback Control Method for Linear Resonant Actuator Using an Estimated External Load from the Back-EMF as a Target Voltage", Proceedings of International Symposium on Power Electronics, Electrical Drive, Automation and Motion, FB4, pp.1547-1551, Jun. 2012.

[11] Yasuyoshi Asai, Katsuhiro Hirata, and Tomohiro Ota, "Amplitude Control Method of Linear Resonant Actuator by Load Estimation from the Back-EMF", IEEE Trans. Magn., VOL.49, NO.5, pp.2253-2256, 2013.05.

[12] T. Yamaguchi, Y. Kawase, H. Kodama, K. Hirata, T. Ota and Y. Hasegawa, "Eddy Current Damping Analysis of Laser Marker Using 3-D Finite Element Method", IEEE Trans. Magn., vol.42, No.4, pp.1011-1014, Apr. 2006.

[13] T. Ota, K. Hirata, T. Yamaguchi, Y. Kawase, K Watanabe and A. Nakase, "Dynamic Response Analysis of Opening and Closing Sensor for Windows", IEEE Trans. Magn., vol.41, No.5, pp.1604-1607, May. 2005.

[14] Satoshi Suzuki, Yoshihiro Kawase, Tadashi Yamaguchi, Katsuhiro Hirata and Yu Okaue, "Dynamic Response Analysis of Shear-Type Compact MR Brake", IEEE Trans. Magn., vol.45, No.3, pp.1-4, Mar. 2009. 